\title{
Elasto-Plasticity Behavior of High Strength Steel Sheet in Biaxial Stress Path Change*
}

\author{
Takeshi Uemori ${ }^{1}$, Tohru Kuramitsu ${ }^{2}$, Yuji Mito², Ryutaro Hino ${ }^{2}$, \\ Tetsuo $\mathrm{Naka}^{3}$ and Fusahito Yoshida ${ }^{2}$ \\ ${ }^{1}$ Department of Mechanical Engineering, Kinki University, Higashi-Hiroshima 739-2116, Japan \\ ${ }^{2}$ Department of Mechanical System Engineering, Graduate School of Engineering, Hiroshima University, \\ Higashi-Hiroshima 739-8527, Japan \\ ${ }^{3}$ Yuge National College of Maritime Technology, Kamijima, Ehime 794-2593, Japan
}

The elasto-plasticity behavior of a high-strength steel sheet of $980 \mathrm{MPa}-\mathrm{TS}$ was investigated by performing biaxial tension experiments. In order to evaluate the accuracy of constitutive models of plasticity in describing such elasto-plasticity behavior, numerical simulations of stressstrain responses were conducted for the same stress paths as those in the experiments using two types of constitutive models: the isotropic hardening model (IH model) and the kinematic hardening model proposed by the present authors (Yoshida-Uemori model). In this work, special emphasis is placed in the cases of stress-path change. In experiments on radial loadings after equi-balanced tension preloading, it was found that flow stresses are considerably lower than uniaxial tension flow stress. The IH model can hardly describe this phenomenon, although it is sufficient for stress-strain analysis of proportional loading cases. In contrast to this, the Yoshida-Uemori model can well predict every stressstrain response in biaxial stress-path changes. [doi:10.2320/matertrans.P-M2010817]

(Received January 31, 2010; Accepted June 21, 2010; Published September 1, 2010)

Keywords: high-strength steel sheet, biaxial tension test, stress path changes, constitutive models of plasticity

\section{Introduction}

High strength steel sheets (HSSs) have been used increasingly in automotive industries to reduce the vehicle fuel consumption and improve the crashworthiness performance. However, a serious technical problem in using HSSs is their poor press-formability, i.e., sheet fracture is more likely to occur during forming and very large springback appears after forming because of possible their thinness due to high strength.

Nowadays, in order to predict the springback and fracture of sheets, forming simulation is conducted widely in pressforming industries. ${ }^{1-4)}$ For the accurate forming simulation, it is of vital importance to use an advanced material model (a constitutive equation of elasto-plasticity) which can describe the complicated elastic-plastic stress-stain responses of sheet metals precisely. Two of the present authors (Yoshida and Uemori) have recently proposed an advanced constitutive model of large-strain cyclic plasticity which can describe cyclic plasticity characteristics, such as the Bauschinger effect and cyclic hardening characteristics, as well as the anisotropy of sheet metals (hereafter, called 'Yoshida and Uemori model' ${ }^{5-7)}$ ). By using this model, the accurate prediction of springback becomes possible. High capability of this model in simulating cyclic behavior has been already verified by comparing the numerical simulations with the various experimental results of uniaxial cyclic tension-compression. However, for multi-axial stress conditions, the accuracy of this model has never been examined, although the constitutive modeling in multi-axial stress state is one of the most important issues since the stress (or

*This paper was Originally Published in Japanese in J. Jpn. Soc. Technol. 50-576 (2009) 39-43. strain) path of a material element changes non-proportionally during press-forming, specifically in multi-stage stamping operation.

In the present research, in order to examine multi-axial plastic deformations, radial loading tests after equi-balanced preloading were performed on a $980 \mathrm{MPa}$ HSS using a biaxial testing machine. These types of non-proportional experiments are quite new compared to other researchers' previous works. For example, Kim and $\mathrm{Yi}^{8)}$ examined the flow stresses in the stress path change but only in the uniaxial stress state for both primary and secondary loadings. Most of biaxial stretching experiments previously reported were on the yield surfaces (equi-plastic work or equi-plastic strain surfaces) determined under proportional loadings.

In the present paper, the performance of Yoshida-Uemori model is discussed by comparing the numerical simulation of such non-proportional stress-strain responses with the corresponding experimental results. Furthermore, the limitation of the isotropic hardening model in simulating the stress-strain behavior under non-proportional loading is described.

\section{Experiment}

\subsection{Material and specimens}

A dual-phase HSS of $980 \mathrm{MPa}-\mathrm{TS}$ of $1.4 \mathrm{~mm}$ thick (made by JFE steel Co., Ltd.) was employed for the experiment. For uniaxial tension experiment type JIS13A specimen was used. Figure 1 shows a cruciform specimen, prepared by laser cutting, for the bi-axial stretching test. The size of gage section in this specimen is $50 \mathrm{~mm} \times 50 \mathrm{~mm}$, where the uniformity of stress distribution in this area was guaranteed by the finite element calculation. The specimen has slits in each arm, which is designed to release the deformation constraint on the gage section. 


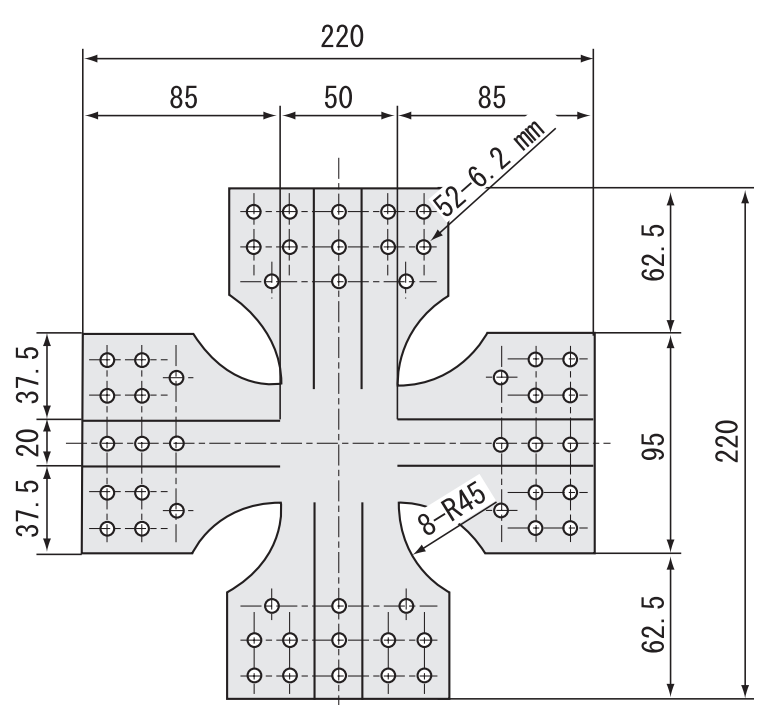

Fig. 1 Biaxial tension test specimen (in $\mathrm{mm}$ ).

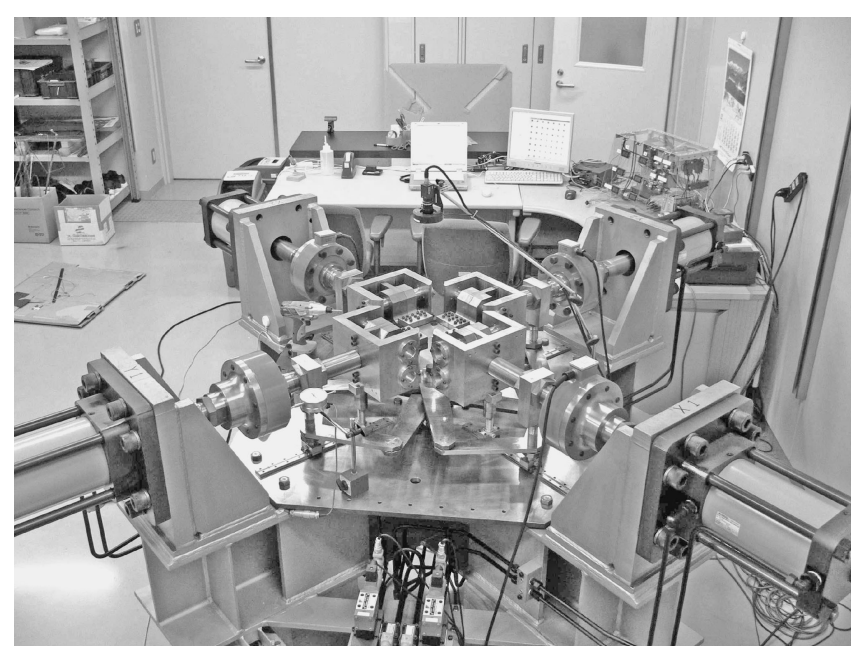

Fig. 2 A photograph of biaxial tension test machine.

\subsection{Biaxial testing machine}

Figure 2 shows a photograph of a servo-controlled hydraulic testing machine, where the cruciform specimen is biaxially stretched in $\mathrm{X}$ (rolling direction of the sheet: R.D.) and Y (transverse direction: T.D.) directions. The load capacity and the maximum stroke of each ram of the machine are $200 \mathrm{kN}$ and $200 \mathrm{~mm}$, respectively. In order to obtain the uniform deformation at the center of the cruciform specimen in the arbitrary multi-axial stress conditions, a feedback load control is applied in the present hydraulic servo system. Tensile loads are measured by load cells, and displacements of the arms of the machine are measured by displacement transducers. Biaxial strains were measured with strain gauges bonded on a surface of the specimen. Besides the strain gauge measurement, the deformations of small circles stamped on the other side of the surface of the specimen were recorded by a CCD camera, and the strains were calculated from them. The difference in strain values obtained from the above two methods was found to be very small, and thus the accuracy of the strain measurement was confirmed.

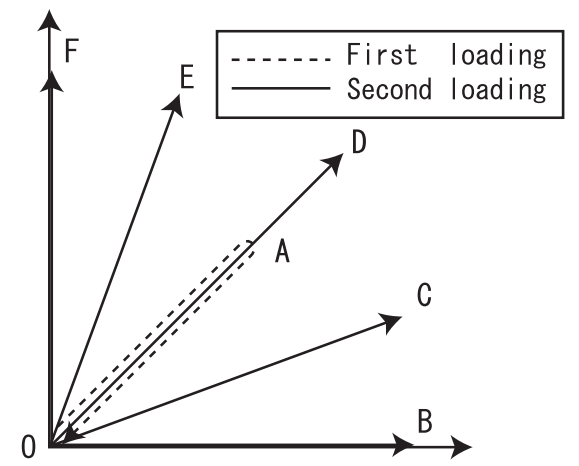

Fig. 3 Schematic illustration of stress path changes. First a specimen is subjected to equi-balanced tension, then full unloading $(\mathrm{O} \rightarrow \mathrm{A} \rightarrow \mathrm{O}$, as shown by broke line), and finally radial loadings $(\mathrm{O} \rightarrow \mathrm{B} \sim \mathrm{F}$, the solid lines).

\subsection{Biaxial tension test}

In order to reveal the plastic deformations of the HSS, biaxial tension tests were conducted under proportional and non-proportional loadings, as schematically illustrated in Fig. 3.

(A) Proportional loading experiment: The specimens were loaded radially in the stress space $\left(\sigma_{x}, \sigma_{y}\right)$ in the following five directions $(\mathrm{O} \rightarrow \mathrm{B}, \mathrm{O} \rightarrow \mathrm{C}, \ldots, \mathrm{O} \rightarrow$ $\mathrm{F}$ in Fig. 3), where stress ratios are $\sigma_{y} / \sigma_{x}=0,1 / 3,1 / 2$, $3 / 4,1,4 / 3,2,3$ and $\infty$. Among these, for uniaxial tensile experiments in $\mathrm{X}$ and $\mathrm{Y}$ directions $\left(\sigma_{y} / \sigma_{x}=0\right.$ in R.D. and $\sigma_{y} / \sigma_{x}=\infty$ in T.D.) type JIS13A specimen were used.

(B) Non-proportional loading experiment: First, equi-balanced stretching $\left(\sigma_{y} / \sigma_{x}=1\right)$ had been imposed on the specimen, and then fully unloaded $(\mathrm{O} \rightarrow \mathrm{A} \rightarrow \mathrm{O}$ in Fig. 3). Next, the specimens were radially loaded again in the following five directions $(\mathrm{O} \rightarrow \mathrm{B}, \mathrm{O} \rightarrow \mathrm{C}, \ldots$, $\mathrm{O} \rightarrow \mathrm{F}$ ), where $\sigma_{y} / \sigma_{x}=0,1 / 2,1,2$, and $\infty$. For $\sigma_{y} / \sigma_{x}=0$ and $\infty$, uniaxial tensile specimens of $\mathrm{X}$ and $\mathrm{Y}$ directions were cut from the pre-strained cruciform specimen.

\section{Analysis}

The aim of the present work is to reveal the deformation characteristics of the HSS under non-proportional biaxial loading by experiment. Furthermore, by comparing thus obtained experimental data of stress-strain responses with the corresponding numerical simulation, the accuracy of constitutive models is discussed. The following two types of models are examined, one is Yoshida-Uemori kinematic hardening model, and the other is classical IH model.

\subsection{Yoshida-Uemori model}

This model has been constructed within the framework of two-surface modeling, wherein the yield surface moves kinematically within a bounding surface, as schematically illustrated in Fig. 4. It describes the Bauschinger effect precisely by an original kinematic hardening law of the yield surface, and furthermore, it can simulate the stress-strain response of workhardening stagnation which appears in most 


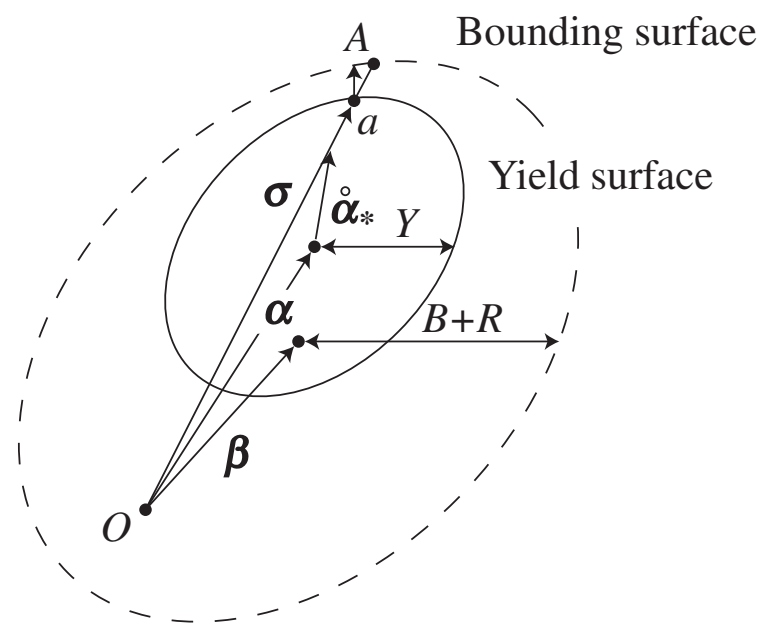

Fig. 4 Schematic illustration of the motion of yield surface within the bounding surface (Yoshida-Uemori model).

of materials in a large reverse deformation. The outline of this model is described as follows.

Since the experimental stress-strain responses under reverse deformation show that the re-yielding starts at a very early stage of stress reversal, we assume only the kinematic hardening for the yield surface, while for the bounding surface mixed isotropic-kinematic hardening. When the yield function at the initial (non-deformed) state, $f_{0}$, has a general form:

$$
f_{0}=\phi(\sigma)-Y=0,
$$

where $\phi$ denotes a function of the Cauchy stress $\sigma$, and $Y$ is the initial yield strength, the subsequent yield function $f$ is given by the equation:

$$
f=\phi(\sigma-\alpha)-Y=0,
$$

where $\boldsymbol{\alpha}$ denote the backstress. The associated flow rule is written as

$$
\dot{\boldsymbol{\varepsilon}}^{p}=\frac{\partial f}{\partial \boldsymbol{\sigma}} \dot{\lambda}
$$

The bounding surface $F$ is expressed by the equation:

$$
F=\phi(\sigma-\beta)-(B+R)=0,
$$

where $\boldsymbol{\beta}$ denotes the center of the bounding surface, and $B$ and $R$ are its initial size and isotropic hardening (IH) component. The relative kinematic motion of the yield surface with respect to the bounding surface is expressed by

$$
\boldsymbol{\alpha}_{*}=\boldsymbol{\alpha}-\boldsymbol{\beta} .
$$

For the evolution of $\boldsymbol{\alpha}_{*}$, we assume

$$
\begin{aligned}
& \dot{\boldsymbol{\alpha}}_{*}=C\left[\left(\frac{a}{Y}\right)(\sigma-\boldsymbol{\alpha})-\sqrt{\frac{a}{\bar{\alpha}}} \boldsymbol{\alpha}_{*}\right] \dot{p}, \\
& \dot{\boldsymbol{\beta}}=m\left[\left(\frac{b}{B+R}\right)(\boldsymbol{\sigma}-\boldsymbol{\beta})-\boldsymbol{\beta}\right] \dot{p}, \\
& \dot{p}=\sqrt{(2 / 3) \dot{\boldsymbol{\varepsilon}}^{p}: \dot{\boldsymbol{\varepsilon}}^{p}}, \bar{\alpha}_{*}=\phi\left(\boldsymbol{\alpha}_{*}\right), \\
& a=B+R-Y,
\end{aligned}
$$

where $b, C$ and $m$ are material constants and $\dot{p}$ is the effective plastic strain rate. To describe the workhardening stagnation,

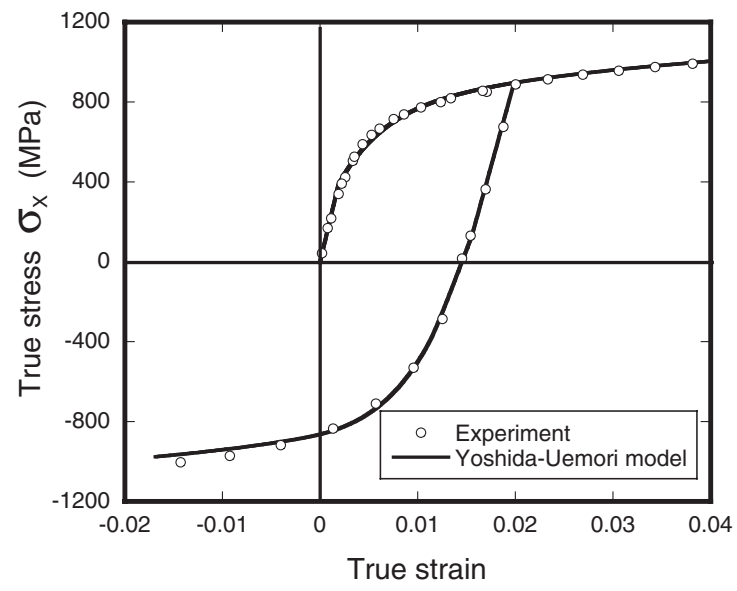

Fig. 5 Stress-strain responses under uniaxial tension and its stress reversal (experimental result and prediction by Yoshida-Uemori model).

a model of non-isotropic-hardening (non-IH) surface, which controls the stagnation of $\mathrm{IH}$ of the bounding surface at a certain range of reverse deformation, is introduced (for details of the modeling, refer to Yoshida et al. ${ }^{5,7)}$ ).

One of the strong features of this model is that it involves limited numbers of material parameters (seven parameters) which can be easily determined from experimental stressstrain responses of uniaxial tension test and the in-plane cyclic tension-compression test (automatic identification of material parameters is possible, e.g., refer to Yoshida et $\left.a l .{ }^{9}\right)$ ). Figure 5 shows the cyclic stress-strain responses of the $980 \mathrm{MPa}$ HSS. The calculated stress-strain responses show a good agreement with the corresponding experimental data. From this figure, we confirmed that this model can describe the Bauschinger effect very well. Our interest and the point of discussion in this paper is how this model can describe the biaxial stress-strain responses properly.

\subsection{Anisotropic yield function}

For the HSS, among the following three yield functions of anisotropy: Hill48, ${ }^{9}{ }^{9} \mathrm{Hill}{ }^{11)}$ and Gotoh, ${ }^{12)}$ an appropriate one was selected by comparing the calculated yield surfaces with the experimental results. From Fig. 6, it was found that the yield surface of the HSS is predicted well by using any of these yield functions. Consequently, we selected the most popular and simplest one, Hill48 quadratic yield function:

$$
\phi=\sqrt{\frac{1}{r_{90}\left(1+r_{0}\right)}\left(r_{90} \sigma_{x}^{2}+r_{0} r_{90} \sigma_{x} \sigma_{y}+r_{0} \sigma_{y}^{2}\right)}
$$

where $r_{0}$ and $r_{90}$ denote the Lankford values for R.D. and T.D., respectively.

\section{Results and Discussion}

\subsection{Equi-plastic strain surfaces under proportional loading}

Figure 7 shows an equi-plastic strain surface obtained from proportional loading experiments, together with the calculated results by the present model (Yoshida-Uemori model incorporating with Hill48 yield function). The 


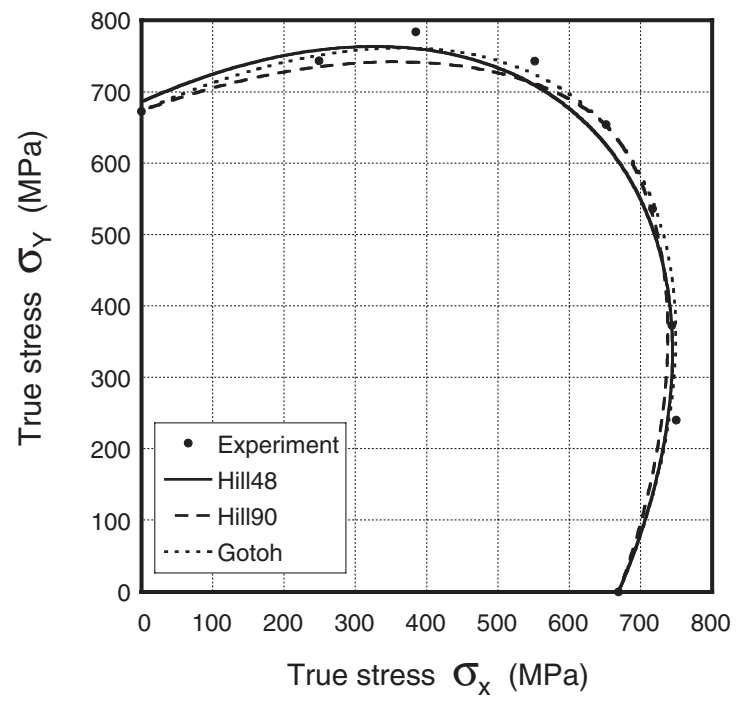

Fig. 6 Experimentally obtained initial yield surface of $980 \mathrm{MPa}$ grade high strength steel sheet, together with the corresponding calculated results by several yield functions; (a) Hill48, (b) Hill90 and (c) Gotoh.

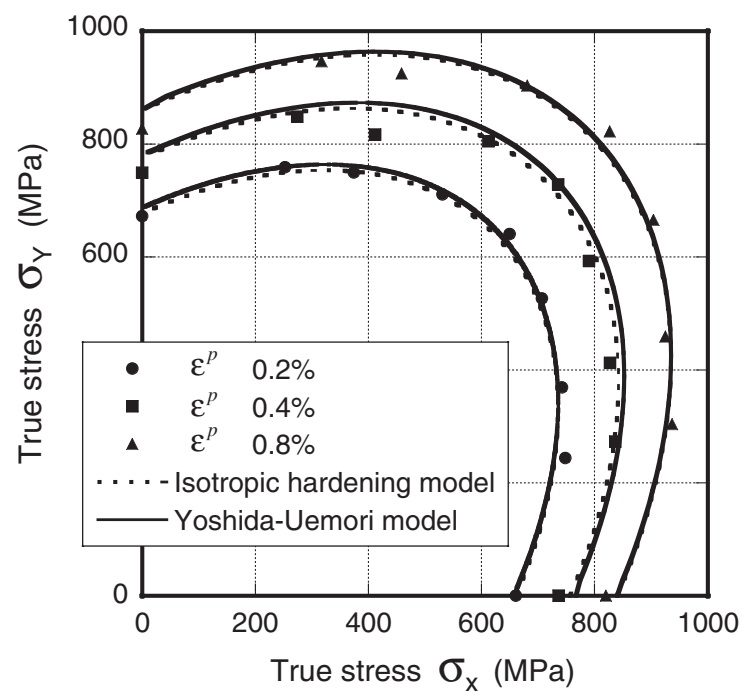

Fig. 7 Equi-plastic strain surfaces obtained in proportional loadings and those numerical predictions by the isotropic hardening model and Yoshida-Uemori kinematic hardening model.

calculated results show a good agreement with the experimental observations. It should be noted that the surfaces calculated by Yoshida-Uemori kinematic hardening model are almost the same as those determined by IH model. This is because the yield surface of Yoshida-Uemori model is approaching the isotropically hardened bounding surface during a monotonic loading.

\subsection{Stress-strain responses in stress-path change}

The plastic strain trajectories in the above experiments of non-proportional stretching $(\mathrm{O} \rightarrow \mathrm{B}, \mathrm{O} \rightarrow \mathrm{C}, \ldots, \mathrm{O} \rightarrow \mathrm{F}$ after preloading-unloading $\mathrm{O} \rightarrow \mathrm{A} \rightarrow \mathrm{O}$ in Fig. 3), as well as the calculated results by Yoshida-Uemori model, are illustrated in Fig. 8. The original point "O" in this figure shows the fully unloaded stress state after pre-straining. Both results agree well with each other.

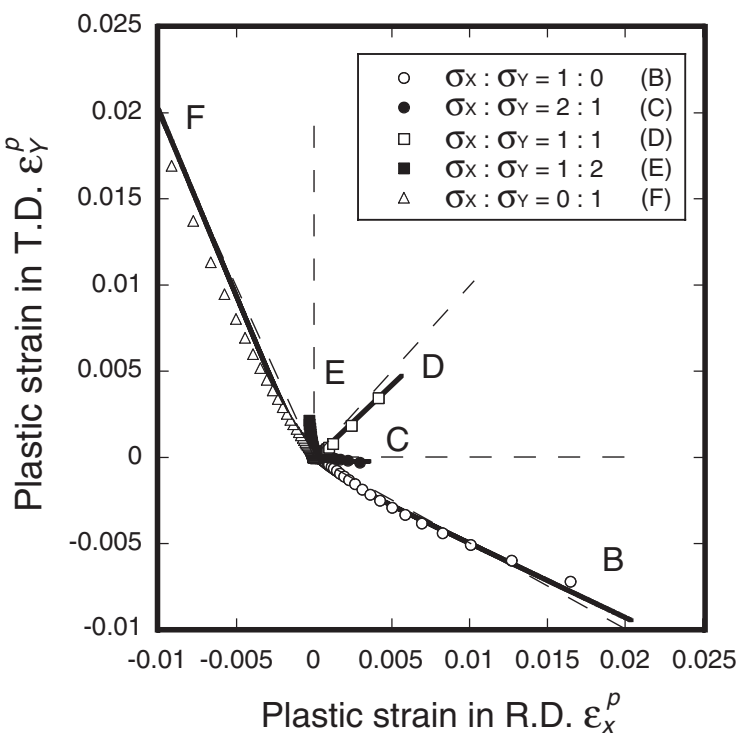

Fig. 8 Plastic strain trajectories in the stress pass change experiments (refer to Fig. 3). Solid line shows the numerical results by YoshidaUemori model and broken line by isotropic hardening model.

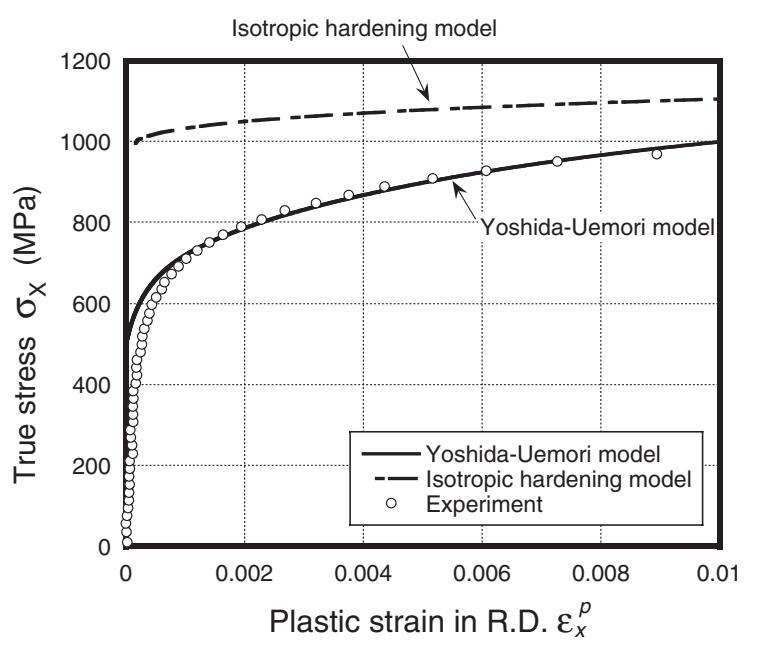

Fig. 9 Stress-plastic strain curve in uniaxial tension after equi-balanced tension preloading.

In contrast to this, IH model completely fails to describe plastic deformations for these stress paths. In the secondary loadings $(\mathrm{O} \rightarrow \mathrm{B}, \mathrm{O} \rightarrow \mathrm{C}, \ldots, \mathrm{O} \rightarrow \mathrm{F})$ no plastic strains are calculated even when applying the same level of stress as one measured in experiment. In the experiment of secondary stressing after biaxial stretching, the plastic flow starts quite early and it progresses under comparatively low stresses. However, IH model hardly describe this phenomenon. The directions of plastic strain rates are calculated by $\mathrm{IH}$ model by applying sufficiently large stresses for plastic deformation. Thus calculated results of the directions (broken lines in Fig. 8) are almost the same as the experimental observations.

From the above discussion, it is concluded that the most serious problem in using IH model is that it hardly predicts the flow stress levels in the stress-path change. Figure 9 shows the stress-plastic strain curve in uniaxial tension after equi-balanced tension preloading (the specimen is stretched 


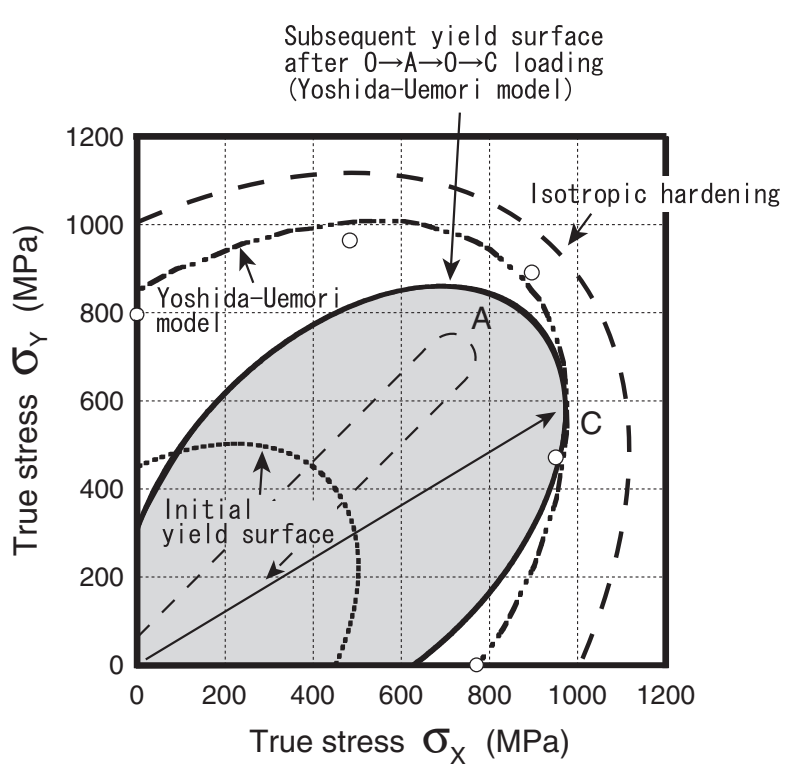

Fig. 10 Equi-plastic strain surface at $0.3 \%$ plastic strain (experimental result $(\bigcirc))$ and model predictions.

in rolling $(\mathrm{X})$ direction of the sheet). Here it should be noted that flow stress of the radial loading after biaxial preloading is significantly lower than uniaxial tension flow stress level, where such a stress-path change would often occur in real sheet stamping operation. Yoshida-Uemori model captures such non-proportional Bauschinger effect very well, whereas IH model completely fails to describe such behavior.

Such non-proportional Bauschinger effect is observed for any secondary radial loading directions. In Fig. 10, the stress points when the effective plastic strain reaches $0.3 \%$ in the secondary loadings are plotted in $\sigma_{x}-\sigma_{y}$ plane. The $0.3 \%$ equi-plastic strain surfaces calculated by Yoshida-Uemori model and IH model are also indicated in this figure. From this, it is found that experimentally obtained equi-plastic strain surface is remarkably smaller than the IH model prediction. Furthermore, the center of the surface is largely shifted to the preloading (equi-balanced biaxial) direction. Yoshida-Uemori model describes such deformation characteristics very well. To demonstrate how the yield surface of Yoshida-Uemori model kinematically moves, the calculated yield surface after a loading path $\mathrm{O} \rightarrow \mathrm{A} \rightarrow \mathrm{O} \rightarrow \mathrm{C}$ is depicted in Fig. 10. From these results it would be concluded that the accurate simulation of the stress-strain responses under non-proportional loading is possible by using the present model.

\section{Conclusions}

Biaxial tension experiments, under proportional and nonproportional loadings, were conducted on $980 \mathrm{MPa}$ HSS. The accuracy of the present authors' constitutive model of plasticity (Yoshida-Uemori model) in simulating stress-strain responses under such non-proportional loadings has been confirmed by comparing the calculated results with the corresponding experimental observations. Some remarks in the present work are as follows.

(1) The flow stress in secondary radial loading after equibalanced preloading is significantly lower than the uniaxial tension flow stress level. Classical IH model hardly describes this phenomenon, although it is sufficient for stress-strain analysis of proportional loading cases.

(2) Yoshida-Uemori model incorporating with Hill48 yield function can well predict every stress-strain response of the HSS in biaxial stress-path change. Thus it is concluded that such an advanced kinematic hardening model is essential for the accurate simulation of stressstrain responses, not only for cyclic behavior but also for non-proportional plastic deformations including stress-strain path change.

\section{REFERENCES}

1) F. Yoshida: Int. J. Plasticity 16 (2000) 359-380.

2) T. Uemori, T. Okada and F. Yoshida: Metals. Mater. 4 (2000) 311-314.

3) K. P. Li, K. W. P. Carden and R. H. Wagoner: Int. J. Mech. Sci. 44 (2002) 103-122.

4) J.-T. Gau, L. K. Gray and L. Kinzel: Int. J. Mech. Sci. 43 (2001) 18131832.

5) F. Yoshida and T. Uemori: Int. J. Plasticity 18 (2002) 661-686.

6) F. Yoshida, T. Uemori and K. Fujiwara: Int. J. Plasticity 18 (2002) 633-659.

7) F. Yoshida and T. Uemori: Int. J. Mech. Sci. 45 (2003) 1687-1702

8) K. H. Kim and J. J. Yin: J. Mech. Phys. Solids 45 (1997) 841-851.

9) R. Hill: Proc. Royal Society of Landon, A 193 (1948) p. 281.

10) F. Yoshida, M. Urabe and V. V. Toropov: Int. J. Mech. Sci. 40 (2003) 237-249.

11) R. Hill: J. Mech. Phys. Solids 38 (1990) 405-417.

12) M. Gotoh: Int. J. Mech. Sci. 19 (1977) 505-512. 